http://jmscr.igmpublication.org/home/ ISSN (e)-2347-176x ISSN (p) 2455-0450

crossref DOI: https://dx.doi.org/10.18535/jmscr/v7i10.47

Journal Of Medical Science And Clinical Research

\title{
Urinary Tract Infection in Pediatric Age Group: A Prospective Disease
}

\author{
Author \\ Dr Ajeet Gopchade [MBBS MD] \\ Consultant Pediatrician, Amrutpath Children Hospital Nanded (MS) \\ *Corresponding Author \\ Dr Ajeet Gopchade [MBBS MD] \\ Consultant Pediatrician, Amrutpath Children Hospital Nanded (MS), India
}

\begin{abstract}
Introduction: Urinary tract infection (UTI) is common in children particularly girls. It usually presents with fever, rigors, urgency and increased frequency of micturition. Its diagnosis depends upon demonstration of sufficient quantity of organisms on microscopic examination and by culture sensitivity. Culture and sensitivity report not only provide with the confirmation of diagnosis but also help in managing the patients. We conducted this study to analyze clinical features and organisms involved in causing UTI in pediatric age group.

Materials and Methods: This was a prospective study in which patients under the age of 12 years. Urine samples from the patients suspected to be having UTI were collected from the patients and was sent for routine microscopic examination as well as for culture sensitivity Pediatric patients with culture proven urinary tract infections were included in this study on the basis of a predefined inclusion and exclusion criteria. Presenting complaints and culture sensitivity reports were analyzed. P value less than 0.05 was taken as statistically significant.

Results: Out of the 30 patients who had been diagnosed with UTI there were 20 females (66.66\%) and 10 males (33.33\%) with a M:F ratio of 1.72. The incidence of UTI was found to be more in female as compared to males and the difference was found to be statistically significant $(P<0.05)$. Most common presenting complaint was found to be fever which was present in all patients (100\%). The other presenting complaints were burning micturition (70\%), abdominal pain (40\%), rigors (36.66\%), increased frequency (36.33\%), urgency (33.33\%), Loin pain (33.33\%) and altered urine color (30\%). Most common offending organism was found to be E. coli (46.67\%) followed by Klebsiella (23.33\%).

Conclusion: Urinary tract infection is one of the common causes of fever in children and must be ruled out in any child presenting with features suggestive of UTI. It's appropriate diagnosis and prompt treatment are crucial to prevent complications such as renal scarring, pyelonephritis and sepsis.

Keywords: Urinary tract infection, Culture and sensitivity, Escherichia coli, antibiotics.
\end{abstract}

\section{Introduction}

Urinary tract infection can be defined as infection of any part of the urinary tract and can be diagnosed on the basis of demonstration of growth of organisms in urine which usually is sterile ${ }^{1}$. It is one of the common causes for which pediatric consultations are sought. Urinary tract infection may have a spectrum which may range from asymptomatic bactiurea to severe infection causing complications such as pyelonephritis and 
septic shock. It usually presents with fever, chills and burning micturition. In infants it may also present with failure to thrive, refusal to feed and irritability $^{2}$. In younger children less than 3 months of age it may present with fever without localizing signs therefor it is important to rule out urinary tract infection in infants presenting with fever without localizing signs. In older children urinary tract infections may present with signs and symptoms such as fever, abdominal pain dysuria, urgency and enuresis. Clinical examination may reveal costovertebral angle tenderness. The criteria for diagnosis of UTI as per American academy of pediatrics is presence of pyuria or bacteriuria on urine analysis and presence of at least 50,000 colony forming units per ml of urine ${ }^{3}$. Other than urine culture complete blood count, blood culture, serum electrolytes and renal functions are needed to be done to rule out complications of urinary tract infection. In selected cases imaging studies such as X-Rau KUB, ultrasonography of abdomen and pelvis and micturating cystourethrography may have to be done $^{4}$. In some cases, nuclear imaging such as DTPa renogram may have to be done to know the effect of UTI on renal functions ${ }^{5}$.

Thecommon organisms responsible for urinary tract infection include Klebsiella, proteus and enterococcus. The uncommon offending agents include pseudomonas, streptococcus B (in neonates) and fungi such as candida. The common risk factors for urinary tract infection include female gender, antibiotic therapy for other infections (by altering periurethralflora), bowel or bladder dysfunction due to any cause, constipation and vesicoureteric reflux ${ }^{6}$. It has been found that uncircumcised children are more susceptible for acquiring UTI as compared to non-circumcised children. A high index of suspicion is needed in these children who are predisposed for developing urinary tract infection because in many children urinary tract infection presents with nonspecific signs and symptoms. Undiagnosed and untreated cases may progress to complications such as renal scarring, hypertension and chronic renal failure ${ }^{7}$.
Once the diagnosis is confirmed the mainstay of treatment is administration of appropriate antibiotics. In uncomplicated UTI where patient is not toxic oral antibiotics and plenty of oral fluids may be advised. Majority of patients with uncomplicated UTI will respond to oral antibiotics dramatically. In cases of complicated UTI and when the patient is toxic an aggressive treatment with parental antibiotics is essential. After the patient responds to parenteral antibiotics patient can be switched to oral antibiotics. A close follow up is required during antibiotic therapy so as to be able to evaluate the response to antibiotics ${ }^{8}$.

We conducted this study to analyze presenting complaints and predisposing factors for development of urinary tract infection in children up to 12 years of age.

\section{Materials and Methods}

This was a prospective study in which children below the age of 12 years were included on the basis of a predefined inclusion and exclusion criteria. The parents or guardian of the patients gave informed written consent to be part of the study. Patients presenting with fever and signs and symptoms suggestive of urinary tract infections were included in this study. A detailed history was taken particularly for the presence of signs and symptoms which could suggest UTI. History of fever, chills and burning micturition was asked and noted down. Urine samples were collected from the patients and was sent for routine microscopic examination as well as for culture sensitivity. In infants urine was collected from sterile urine bag attached to genitalia whereas in older children mid-stream clean catch urine sample was collected after washing the genitalia with soap and water. Suprapubic aspiration, being an invasive procedure, was not attempted in any of the children presenting with signs and symptoms suggestive of urinary tract infection. Presence of more than $10^{5}$ colony forming units of a single organism was taken as urinary tract infection. Complete blood count and renal function tests were also sent. Patients were treated 
by appropriate oral or parenteral antibiotics depending upon the severity of the urinary tract infection. In cases where urine culture turned out to be positive antibiotics were switched on the basis of susceptibility pattern of the culture sensitivity report. In patients who had been started with parenteral antibiotics the switch over to oral antibiotics was done once the fever has subsided and signs and symptoms of UTI is reduced. For the purpose of statistical analysis SSPS 21.0 software was used. Microsoft excel and word was used for statistics and graphs preparation. $\mathrm{P}$ value less than 0.05 was taken as statistically significant.

\section{Inclusion Criteria}

1- Patients presenting with signs and symptoms suggestive of urinary tract infection and diagnosed to be having UTI on the basis of positive culture report.

2- Age of the patient below 12 years.

3- Parents or guardians gave informed written consent

\section{Exclusion Criteria}

1- Those who refused consent.

2- Age more than 12 years.

3- Patients with pre-existing renal diseases such as nephrotic syndrome, renal failure of obstructive uropathy.

\section{Results}

During the study period 180 patients presented to pediatric Outpatient department with history of fever and signs and symptoms on the basis of which UTI was suspected. Out of these 180 patients the diagnosis of urinary tract infection was confirmed in 30 patients on the basis of culture and sensitivity test. Out of the 30 patients who had been diagnosed with UTI there were 20 females $(66.66 \%)$ and 10 males $(33.33 \%)$ with a $\mathrm{M}: \mathrm{F}$ ratio of 1.72 .

\section{UTI IN STUDIED CASES}

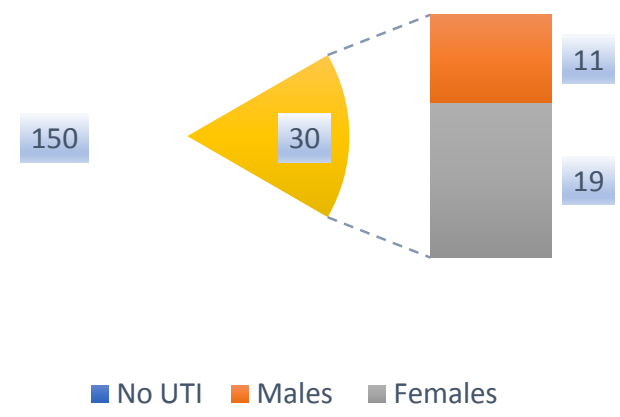

Figure 1: Gender wise distribution of cases

Out of the total patients presenting with fever and suspected to be having UTI there were 96 males and 84 females. Out of 96 males with suspected UTI 10 patients were confirmed to be having UTI on the basis of culture and sensitivity reports whereas amongst 84 females 20 were found to have UTI on the basis of culture sensitivity report. The incidence of UTI was found to be more in female as compared to males and the difference was found to be statistically significant $(\mathrm{P}<0.05)$.

Table 1: Age Distribution of the studied cases

\begin{tabular}{|l|c|c|c|}
\hline & $\begin{array}{c}\text { Number of } \\
\text { patients with } \\
\text { suspected UTI }\end{array}$ & No UTI & $\begin{array}{c}\text { Confirmed } \\
\text { UTI } \\
\text { (culture } \\
\text { positive) }\end{array}$ \\
\hline Males & 96 & 86 & 10 \\
\hline Females & 84 & 64 & 20 \\
\hline Total & 110 & 150 & 30 \\
\hline $\mathbf{P}=\mathbf{0 . 0 0 3}$ (Significant)
\end{tabular}

The analysis of the age groups of the culture positive UTI cases showed that the most common affected age group was between 2-5 years (43.33\%) Followed by 5-8 years $(23.33 \%)$ and 8 10 years $(16.67 \%)$.

Table 2: Age group of the affected cases

\begin{tabular}{|l|c|c|}
\hline Age Group & No of Cases & Percentage \\
\hline$<2$ Years & 2 & $6.67 \%$ \\
\hline $2-5$ Years & 13 & $43.33 \%$ \\
\hline $5-8$ Years & 7 & $23.33 \%$ \\
\hline $8-10$ Years & 5 & $16.67 \%$ \\
\hline$>10$ years & 3 & $10.00 \%$ \\
\hline Total & 30 & $100 \%$ \\
\hline
\end{tabular}


The analysis of presenting complaints of the studied cases showed that the most common presenting complaint in studied cases was fever which was present in all 30 cases $(100 \%)$. The other common complaints included burning micturition which was present in $21(70 \%)$ patients, abdominal pain (40\%), rigors $(36.66 \%)$, increased frequency $(36.33 \%)$, urgency $(33.33 \%)$, Loin pain $(33.33 \%)$ and altered urine color $(30 \%)$.

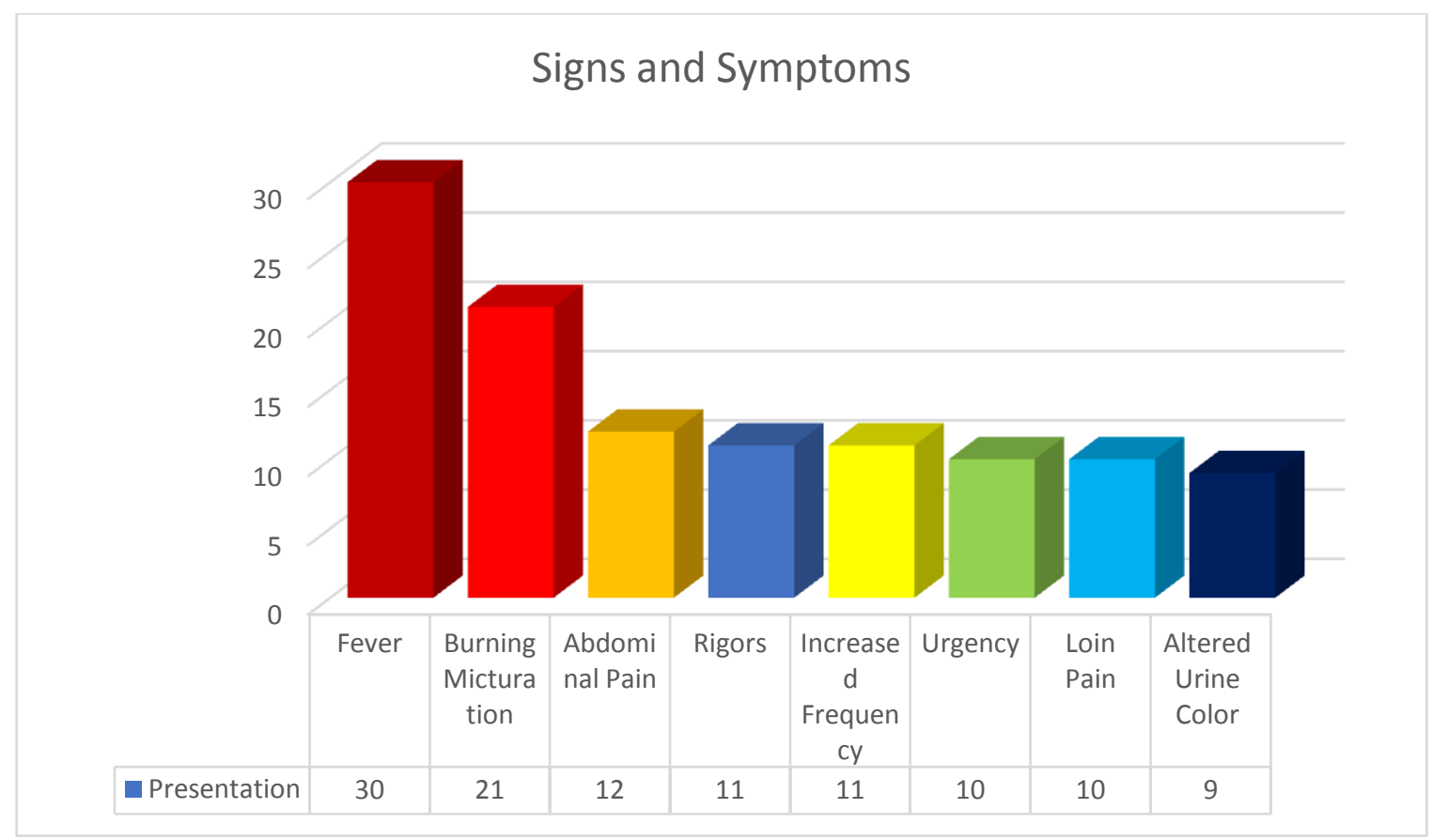

Figure 2: Presenting Complaints in the studied cases.

The analysis of cases on the basis of culture and sensitivity reports showed that the most offending organism in cases of urinary tract infection was Escherichia coli $(46.67 \%)$ followed by klebsiella $(23.33 \%)$ and enterococcus faecalis (10\%). The other less common organism responsible for UTI were found to be P. aeruginosa $(6.67 \%)$ and coagulase negative staph aureus (3.33\%). In 3 patients polymicrobial growth was seen.

Table 3: Organisms isolated on urine culture

\begin{tabular}{|l|c|c|}
\hline Offending Organism & No Of Cases & Percentage \\
\hline Escherichia coli & 14 & $46.67 \%$ \\
\hline klebsiella & 7 & $23.33 \%$ \\
\hline enterococcus faecalis & 3 & $10.00 \%$ \\
\hline P.aeruginosa & 2 & $6.67 \%$ \\
\hline $\begin{array}{l}\text { coagulase negative staph } \\
\text { aureus }\end{array}$ & 1 & $3.33 \%$ \\
\hline Polymicrobial Growth & 3 & $10.00 \%$ \\
\hline Total & 30 & $100 \%$ \\
\hline
\end{tabular}

\section{Discussion}

Urinary tract infection is one of the common causes for which pediatric consultations are sought. Since urinary tract infection presents with non-specific signs and symptoms it is important that a high index of suspicion is maintained particularly in younger children in whom UTI may manifest with non-specific signs and symptoms.

In our study females were more commonly affected than males and the difference was found to be statistically significant. Many studies have reported that urinary tract infection is more common in females than males. Mendez SV et al conducted a Retrospective review over a period of one year of children with culture positive UTI in pediatric patients. A total of 40 children were included in the study. Majority of study subjects were females in the age group of 1-5 years $(60 \%)$. Most common symptoms reported in children $<5$ years was fever $(58 \%)$ and excessive cry while passing urine $(58 \%)$ whereas $50 \%$ of children $>5$ years had complaints of pain abdomen. Previous history of UTI was present in $22 \%$ of subjects. Urine analysis had a sensitivity of $75 \%$ and 
specificity of $67 \%$. The organism predominantly causing UTI were found to be E. coli $(67 \%)^{9}$. Female predominance in cases of UTI was also reported by the authors such as Kumar A et al and et $\mathrm{al}^{10}$ and Jayavarthinni $\mathrm{M}$ et $\mathrm{al}^{11}$.

In our study the most common presenting complaint was found to be fever which was present in all patients $(100 \%)$. The other common presenting complaints included burning micturition which was present in 21 (70\%) patients, abdominal pain $(40 \%)$, rigors $(36.66 \%)$, increased frequency (36.33\%), urgency (33.33\%), Loin pain $(33.33 \%)$ and altered urine color $(30 \%)$. Zeno LF conducted a cross sectional study to know about the clinical and microbiological profile of urinary tract infection. Out of the 40 patients studied, fever (85\%) was the most common presenting symptom and Escherichia coli $(57.50 \%)$ was the most common organism isolated followed by Klebsiella (15\%). The most sensitive antibiotic was meropenem $(75 \%)$ and the most resistant antibiotic was cefazolin (87.50\%). These results were found to be statistically significant ${ }^{12}$. Fever was the common presenting complaint in patients in UTI also in the studies conducted by Rowe TA et $\mathrm{al}^{13}$ and Ismaili $\mathrm{K}$ et $\mathrm{al}^{14}$.

In our study E. coli was the most common offending organism $(46.67 \%)$ isolated on the basis of culture and sensitivity reports. The other common organisms involved were klebsiella $(23.33 \%)$ and enterococcus faecalis (10\%). The other less common organism responsible for UTI were found to be P.aeruginosa(6.67\%) and coagulase negative staph aureus (3.33\%). In 3 patients polymicrobial growth was seen. Angmo $\mathrm{D}$ et al conducted a cross sectional study in which urine sample from 2100 patients with clinical symptoms of UTI were collected. The authors found that Escherichia coli 114(57.3\%) was the predominant organism followed by Klebsiella pneumoniae $22(11.1 \%)$ and enterococcus fecalis $19(9.5 \%)$. E. coli resistance to various antibiotics were as follows, cotrimoxazole (67.5\%), norfloxacin (66.6\%), cefoperazone/ sulbactam $\quad(57 \%), \quad$ piperacillin/tazobactam
(50.8\%), nitrofurantoin (35\%), ertapenem (33.3\%), gentamicin and meropenem (32.4\%), ciprofloxacin (23.6\%) imipenem (14\%) and amikacin $(13.1 \%)^{15}$.

\section{Conclusion}

Urinary tract infection is one of the common causes for which pediatrics consultation is sought. Its early diagnosis and appropriate management is essential to prevent further complications. Gram negative organisms particularly E.Coli and Klebsiella are common offending organisms in our study.

\section{Conflict of Interest: None}

\section{References}

1. Schmiemann G, Kniehl E, Gebhardt K, Matejczyk MM, Hummers-Pradier E. The diagnosis of urinary tract infection: a systematic review. Dtsch Arztebl Int. 2010;107(21):361-367.

2. Najar MS, Saldanha CL, Banday KA. Approach to urinary tract infections. Indian J Nephrol. 2009;19(4):129-139.

3. Doern CD, Richardson SE. Diagnosis of Urinary Tract Infections in Children. J Clin Microbiol. 2016;54(9):2233-2242.

4. Riccabona M. Imaging in childhood urinary tract infection. Radiol Med. 2016 May;121(5):391-401.

5. Madsen CJ, Møller ML, Zerahn B, Fynbo C, Jensen JJ. Determination of kidney function with 99mTc-DTPA renography using a dual-head camera. Nucl Med Commun.2013 Apr;34(4):3227.

6. Mattoo TK. Vesicoureteral reflux and reflux nephropathy. Adv Chronic Kidney Dis. 2011;18(5):348-354.

7. Park YS. Renal scar formation after urinary tract infection in children. Korean J Pediatr. 2012;55(10):367-370.

8. Kang CI, Kim J, Park DW, et al. Clinical Practice Guidelines for the Antibiotic 
Treatment of Community-Acquired Urinary Tract Infections. Infect Chemother 2018;50(1):67-100.

9. Sharon Victoria Mendez, Jaidev M.D, Pavan Hegde, Habeeb Ullah Khan Clinical, Bacteriological Profile and Management Practices in Culture Positive Urinary Tract Infection in Children JMSCR Volume 06 Issue 12 December 2018 630-634

10. Ashit Kumar, Ghazi S Ahmad, Kumar S Nath, Shreshy Singh, MD Arshad Alam Prevalence of urinary tract pathogen and its antimicrobial susceptibility pattern in children admitted in $\mathrm{KMCH}$, Katihar, Bihar and compare its susceptibility pattern from national trend JMSCR Volume 06 Issue 10 October 2018 p 942946

11. Jayavarthinni M, Madan S. Urinary Tract Infection among Inpatients: An Insight into Risk Factors and Antimicrobial Resistance. Ann. Int. Med. Den. Res. 2018; 4(2): MB01-MB05.

12. Dr J. Louis Ferdin Zeno, Dr M. K. Uthaya Sankar, Dr R. Gopal, Dr I. S. Suman Babu Study of Clinical profile and Antibiotic susceptibility of Urinary Tract Infection in a Tertiary Care Hospital JMSCR Volume 07 Issue 08 August 2019 342-351.

13. Rowe TA, Juthani-Mehta M. Diagnosis and management of urinary tract infection in older adults. Infect Dis Clin North Am. 2014 Mar;28(1):75-89.

14. Ismaili K, Wissing KM, Lolin K, Le PQ, Christophe C, Lepage P, Hall M. Characteristics of first urinary tract infection with fever in children: a prospective clinical and imaging study. Pediatr Infect Dis J. 2011 May;30(5):3714.

15. Dr Dekyong Angmo, Dr Shaista Nazir, Dr Bashir A Fomda, Dr Shadan Akhtar Dr Shazia Benazir, Dr Asifa Bhat, Dr Leenah
Bashir Microbiological Profile of Urinary Tract infections among Patients attending a Tertiary care Hospital JMSCR Volume 06 Issue 06 June 2018 p 298-303. 